\title{
Numerical study on saturated pool boiling heat transfer in presence of a uniform electric field using lattice Boltzmann method
}

\author{
Yuan Feng ${ }^{a}$, Huixiong Li $^{\mathrm{a}, *}$, Kaikai Guo ${ }^{a}$, Xianliang Lei ${ }^{\mathrm{a}}$, Jianfu Zhao ${ }^{\mathrm{b}, \mathrm{c}}$ \\ a State Key Laboratory of Multiphase Flow in Power Engineering, Xi'an Jiaotong University, Xi'an 710049, China \\ ${ }^{\mathrm{b}}$ CAS Key Laboratory of Microgravity, Institute of Mechanics, Chinese Academy of Sciences, Beijing 100190, China

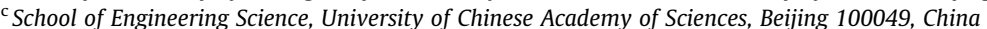

\section{A R T I C L E I N F O}

Article history:

Received 3 November 2018

Received in revised form 23 January 2019

Accepted 27 January 2019

\section{Keywords:}

Lattice Boltzmann method

Electrohydrodynamics

Critical heat flux

Saturated pool boiling

Boiling curves

\begin{abstract}
A B S T R A C T
For the industrial equipment with boiling, critical heat flux (CHF) is an important parameter that determines its upper limit of heat load for safe operation. Therefore, increasing CHF is of great importance in engineering field. Previous studies proved that it's an effective way to enhance boiling heat transfer and CHF by electrohydrodynamics (EHD). However, it is difficult to investigate the influence mechanism of the electric field on the bubble dynamics and boiling heat transfer because of the limit of experimental technical methods. Thus, in this paper, a two-dimensional lattice Boltzmann model was established by coupling the pseudopotential model with phase-change model and electric field model to meet the lack of studies on simulating the pool boiling in presence of an electric field. After validating the rationality of the two-dimensional lattice Boltzmann model established at present study, the heat transfer during nucleate boiling and film boiling under a uniform electric field were studied in detail. In addition, the influence of electric field intensity on boiling curves and CHF was investigated quantitatively. In the partial nucleate boiling regime, a uniform electric field had limited influence on bubble dynamics and boiling heat transfer. In the fully developed nucleate boiling regime, increasing electric field intensity could enhance boiling heat transfer obviously. In the film boiling regime, increasing electric field intensity could slightly enhance averaged heat flux at first. After the electric field intensity reached a certain value, averaged heat flux increased rapidly. Increasing electric field intensity could expand the nucleate boiling regime, and enhance CHF and the wall temperature at CHF point.
\end{abstract}

(c) 2019 Elsevier Ltd. All rights reserved.

\section{Introduction}

As an efficient way of heat transfer, boiling can be commonly observed in many industrial applications, such as nuclear reactors, computer chips and steam generators. It is a common knowledge that the pool boiling under a given wall temperature could be divided into three stages [1]. Concretely, with an increase in wall superheat, pool boiling will fall into the nucleate boiling regime, transition boiling regime and film boiling regime, successively. Critical heat flux (CHF) is an important parameter which sets the upper limit of fully developed nucleate boiling for safe operation of equipment. For the pool boiling process under the heating conditions with controlled heat flux, once the given heat flux exceeds $\mathrm{CHF}$, heat transfer deterioration will take place. Thus, improving $\mathrm{CHF}$ is of great importance in engineering.

\footnotetext{
* Corresponding author.

E-mail address: huixiong@mail.xjtu.edu.cn (H. Li).
}

With the advantages of simple equipment and low power consumption, it's easy to control bubble dynamics and to enhance the boiling heat transfer efficiency via Electrohydrodynamics (EHD). Therefore, it's an efficient method to enhance boiling heat transfer and improve CHF by EHD. In the early years, experimental method is the main method to study the bubble dynamics and heat transfer characteristics during pool boiling under the effect of EHD [2-6]. However, some details during pool boiling, such as the distribution of temperature around the bubble, were difficult to obtain in experimental studies. Numerical method is a good way to solve this problem.

Using traditional CFD methods, the effect of EHD on bubble dynamics [6-9] during nucleate boiling or film boiling by some researchers. Using the level set method, Welch and Biswas [7] concluded that increasing electric field intensity could reduce wall temperature at given heat flux condition during film boiling. Using the CLSVOF algorithm, Tomar et al. [8] found that the bubble shape and ebullition cycle can be modified by strong electric fields during film boiling. Pandey et al. [9] also utilized CLSVOF algorithm to 


\begin{tabular}{|c|c|c|c|}
\hline \multicolumn{4}{|c|}{ Nomenclature } \\
\hline$a, b, \omega$ & parameters in Peng-Robinson EOS & $T_{\text {sat }}$ & saturation temperature, $\mathrm{K}$ \\
\hline $\mathrm{Bo}_{e}{ }^{*}$ & electric Bond number & $T_{w}$ & outer wall temperature, $\mathrm{K}$ \\
\hline$c_{v}$ & specific heat at constant volume, $\mathrm{J} /(\mathrm{kg} \cdot \mathrm{K})$ & $u, \mathbf{u}$ & fluid velocity, $\mathrm{m} / \mathrm{s}$ \\
\hline$D_{d}$ & bubble departure diameter, $\mathrm{m}$ & $V$ & electric potential, $\mathrm{V}$ \\
\hline $\mathbf{e}_{\alpha}$ & lattice velocity vector & $w_{\alpha}$ & weighting coefficient \\
\hline $\mathbf{E}$ & electric field intensity, $\mathrm{N} / \mathrm{C}$ & $\mathbf{x}$ & position \\
\hline$E_{0}$ & characteristic electric field intensity, N/C & & \\
\hline$f_{\alpha}, \mathbf{f}$ & distribution function for density & \multicolumn{2}{|c|}{ Greek symbols } \\
\hline $\mathbf{F}$ & external force, $\mathrm{N}$ & $\Delta T$ & wall superheat, $\mathrm{K}$ \\
\hline$F_{\alpha}^{\prime}$ & forcing term in the velocity space & $\Delta t$ & time step, $s$ \\
\hline $\mathbf{F}_{\text {ads }}$ & fluid-solid interaction force, $\mathrm{N}$ & $\tau$ & relaxation time \\
\hline $\mathbf{F}_{\mathrm{e}}$ & electric force, $\mathrm{N}$ & $\Lambda$ & diagonal matrix of relaxation time \\
\hline $\mathbf{F}_{\mathrm{g}}$ & gravity, $\mathrm{N}$ & $\rho$ & density, $\mathrm{kg} / \mathrm{m}^{3}$ \\
\hline $\mathbf{F}_{\mathrm{m}}$ & Intermolecular interaction force, $\mathrm{N}$ & $v$ & kinematic coefficient of viscosity, $\mathrm{m}^{2} / \mathrm{s}$ \\
\hline g & gravitational acceleration, $\mathrm{kg} \cdot \mathrm{m} / \mathrm{s}^{2}$ & $\sigma$ & parameter to tune the mechanical stability \\
\hline G & interaction strength between fluid & $\psi$ & pseudopotential \\
\hline$G_{w}$ & fluid-solid interaction strength & $\lambda$ & thermal conductivity, $\mathrm{W} /(\mathrm{m} \cdot \mathrm{K})$ \\
\hline$h$ & heat transfer coefficient & $\varepsilon_{0}$ & the permittivity of vacuum, $\mathrm{F} / \mathrm{m}$ \\
\hline$h_{f g}$ & latent heat of vaporization, $\mathrm{J} / \mathrm{kg}$ & $\varepsilon$ & the relative permittivity of fluid \\
\hline Ja & Jacob number & $\Pi_{\alpha}$ & distribution function for electric potential \\
\hline$L_{x}, L_{y}$ & width and length of computational domain, $\mathrm{m}$ & $\chi$ & thermal diffusion coefficient, $\mathrm{m}^{2} / \mathrm{s}$ \\
\hline M & orthogonal transformation matrix & $\gamma$ & surface tension, $\mathrm{N} / \mathrm{m}$ \\
\hline$p_{\text {EOS }}$ & prescribed non-ideal equation of state & & \\
\hline$q(t)$ & space-averaged heat flux & \multicolumn{2}{|c|}{ Subscripts and superscripts } \\
\hline$q_{w}$ & space- \& time-averaged heat flux & & critical properties \\
\hline$R_{d}$ & radius of bubble/droplet, m & $e q$ & equilibrium properties \\
\hline$s(\mathbf{x})$ & switch function & $L, V$ & liquid, vapor \\
\hline $\mathbf{S}$ & forcing term in the moment space & $s$ & $\begin{array}{l}\text { solid } \\
\text { solu, vapoi }\end{array}$ \\
\hline$t$ & time, $\mathrm{s}$ & $x y$ & direction \\
\hline$T$ & temperature, $\mathrm{K}$ & $x, y$ & lattice direction \\
\hline$T_{b}$ & inner wall temperature, $\mathrm{K}$ & $\alpha$ & \\
\hline
\end{tabular}

study the effect of a uniform electric field on film boiling under different gravities, and they concluded that the influence of electric field intensity on averaged Nusselt number will be stronger during film boiling under microgravity. However, bubble nucleation couldn't be simulated by traditional CFD methods. Therefore, it's hard to obtain boiling curves and study EHD's effect on boiling curves and CHF using traditional CFD methods.

Recently, the lattice Boltzmann method (LBM) has been applied to simulate liquid-vapor phase change. Generally, the existing LB models for liquid-vapor phase change can be divided into two categories [10]. The first category is based on the phase-field LB method. Using this method, the phase interface is captured by solving the interface-capturing equation ( $\mathrm{C}-\mathrm{H}$ equation) and the phase change is realized by adding a source term into the continuity equation or the $\mathrm{C}-\mathrm{H}$ equation. Many scholars based on this method to simulate the pool nucleate boiling or film boiling [11-16]. For instance, Sadeghi et al. [12,13] studied the bubble dynamics and heat transfer during saturated nucleate boiling and film boiling using three-dimensional (3D) LB model.

The second category to simulate liquid-vapor phase change is based on the pseudopotential LB model [17]. Using this method, the entire boiling processes, including bubble nucleation process, could be simulated. Gong and Cheng $[18,19]$ used this method to simulate the pool boiling process under different wall superheats and contact angles, and they found that CHF occurs at a lower degree of superheat on a hydrophobic surface than on a hydrophobic surface. Ma et al. [20] found that CHF occurs at a lower wall superheat and the CHF is lower under microgravity conditions than that under the normal gravity. Li et al. $[10,21]$ simulated the pool boiling under different wall superheats and contact angles using multi-relaxation-time (MRT) pseudopotential LB model and found that increasing the contact angle could enhance the onset of boiling. However, few scholars used this model to investigate the effect of EHD on pool boiling heat transfer and the rationality of this model to simulate the pool boiling under an electric field hadn't been proved.

To solve these problems, a two-dimensional LB model was developed in this paper to simulate the effect of EHD pool boiling by coupling the MRT pseudopotential LB model with phase-change model and electric field model. The boiling processes under different wall superheats and electric field intensities were studied in detail, and the effect of electric field intensity on boiling curves and CHF was investigated numerically for the first time. Our research work provided a new method to study the influence of EHD on boiling heat transfer.

\section{Numerical methods}

\subsection{Two-phase flow model}

The distribution of phase field and flow field during pool boiling is solved by MRT pseudopotential lattice Boltzmann model. The lattice Boltzmann equations with a MRT collision operator [10] can be expressed as Eq. (1).

$f_{\alpha}\left(\mathbf{x}+\mathbf{e}_{\alpha} \Delta t, t+\Delta t\right)=f_{\alpha}(\mathbf{x}, t)-\left(\mathbf{M}^{-1} \Lambda \mathbf{M}\right)_{\alpha \beta}\left(f_{\beta}-f_{\beta}^{e q}\right)+\Delta t F_{\alpha}^{\prime}$

where $f_{\alpha}$ is the density distribution function, $f_{\alpha}^{e q}$ is its equilibrium distribution function. $\mathbf{M}$ is an orthogonal transformation matrix, $\mathbf{e}_{\alpha}$ is the discrete velocity at the $\alpha^{\text {th }}$ direction. For D2Q9 model used 
at present study, the expressions of $\mathbf{M}$ and $\mathbf{e}_{\alpha}$ can be found in Ref. [22]. $\boldsymbol{\Lambda}$ is a diagonal matrix that consists of relaxation times which can be expressed as Eq. (2). $F_{\alpha}{ }^{\prime}$ is the forcing term in the velocity space [23], which can be expressed as Eq. (3).

$\mathbf{\Lambda}=\operatorname{diag}\left(\tau_{\rho}^{-1}, \tau_{e}^{-1}, \tau_{\varsigma}^{-1}, \tau_{j}^{-1}, \tau_{q}^{-1}, \tau_{j}^{-1}, \tau_{q}^{-1}, \tau_{v}^{-1}, \tau_{v}^{-1}\right)$

$F_{\alpha}^{\prime}=w_{\alpha}\left(1-\frac{1}{2 \tau_{v}}\right)\left[\frac{\mathbf{e}_{\alpha} \cdot \mathbf{F}}{c_{s}^{2}}+\frac{(\mathbf{u} \mathbf{F}+\mathbf{F u}):\left(\mathbf{e}_{\alpha} \mathbf{e}_{\alpha}-c_{s}^{2} \mathbf{I}\right)}{2 c_{s}^{4}}\right]$

where $w_{\alpha}$ are the weights. For the D2Q 9 model, $w_{0}=4 / 9 ; w_{\alpha}=1 / 9$ when $\alpha=1-4 ; w_{\alpha}=1 / 36$ when $\alpha=5-8$. $\mathbf{u}$ is the macroscopic velocity and $|\mathbf{u}|=\sqrt{u_{x}^{2}+u_{y}^{2}}$. $\mathbf{F}$ is the external force, containing intermolecular interaction force $\mathbf{F}_{\mathrm{m}}$, fluid-solid interaction force $\mathbf{F}_{\mathrm{ads}}$, the buoyant force $\mathbf{F}_{\mathrm{g}}$ and electric field force $\mathbf{F}_{\mathrm{e}}$.

Using the transformation matrix M, Eq. (1) can be rewritten [10] as follows.

$\mathbf{m}\left(\mathbf{x}+\mathbf{e}_{\alpha} \Delta t, t+\Delta t\right)=\mathbf{m}(\mathbf{x}, t)-\boldsymbol{\Lambda}\left(\mathbf{m}-\mathbf{m}^{e q}\right)+\Delta t\left(\mathbf{I}-\frac{\boldsymbol{\Lambda}}{2}\right) \mathbf{S}$

where $\mathbf{m}=\mathbf{M f}, \mathbf{m}^{e q}=\mathbf{M} \mathbf{f}^{\text {eq }}$. $\mathbf{m}^{\text {eq }}$ can be calculated by Eq. (5). $\mathbf{S}$ is the forcing term in moment space [24] and can be expressed as Eq. (6).

$\mathbf{m}^{e q}=\rho\left(1,-2+3|\mathbf{u}|^{2}, 1-3|\mathbf{u}|^{2}, u_{x},-u_{x}, u_{y},-u_{y}, u_{x}^{2}-u_{y}^{2}, u_{x} u_{y}\right)^{T}$

$\mathbf{S}=\left[0,6 \mathbf{u} \cdot \mathbf{F}+\frac{\sigma\left|\mathbf{F}_{m}\right|^{2}}{\psi^{2} \Delta t\left(\tau_{e}-0.5\right)},-6 \mathbf{u} \cdot \mathbf{F}-\frac{\sigma\left|\mathbf{F}_{m}\right|^{2}}{\psi^{2} \Delta t\left(\tau_{\zeta}-0.5\right)}\right.$,

$\left.F_{x},-F_{x}, F_{y},-F_{y}, 2\left(u_{x} F_{x}-u_{y} F_{y}\right), u_{x} F_{y}+u_{y} F_{x}\right]^{2}$

Macroscopic density and velocity [25] are calculated by Eq. (7). $\mathbf{F}_{\mathrm{m}}, \mathbf{F}_{\mathrm{ads}}$ and $\mathbf{F}_{\mathrm{g}}$ can be calculated by Eqs. (8)-(10).

$\rho=\sum_{\alpha} f_{\alpha}, \rho \mathbf{u}=\sum_{\alpha} \mathbf{e}_{\alpha} f_{\alpha}+\frac{\Delta t}{2} \mathbf{F}$

$\mathbf{F}_{m}=-3 G \psi(\mathbf{x}) \sum_{\alpha=1}^{8} w_{\alpha} \psi\left(\mathbf{x}+\mathbf{e}_{\alpha}\right) \mathbf{e}_{\alpha}$

$\mathbf{F}_{\mathrm{ads}}=-G_{w} \psi(\mathbf{x}) \sum_{\alpha=1}^{8} w_{\alpha} \psi(\mathbf{x}) s\left(\mathbf{x}+\mathbf{e}_{\alpha}\right) \mathbf{e}_{\alpha}$

$\mathbf{F}_{\mathrm{g}}=\left(\rho-\rho_{\text {ave }}\right) \mathbf{g}$

In Eq. (8), $G$ is the intermolecular interaction strength and $\psi$ is the pseudopotential that is expressed as Eq. (11). In Eq. (9), $G_{w}$ is fluid-solid interaction strength that is utilized to tune the contact angle. $s\left(\mathbf{x}+\mathbf{e}_{\alpha}\right)$ is the switch function that equals to 1 and 0 for solid or fluid phase, respectively. In Eq. (10), $\mathbf{g}=\left(0,-g_{y}\right)$ is the gravitational acceleration and $\rho_{\text {ave }}$ is the average density in the computational domain.

$\psi(\mathbf{x})=\sqrt{2\left|\left(p_{E O S}-\frac{1}{3} \rho\right) / G\right|}$

where $p_{\mathrm{EOS}}$ is the prescribed non-ideal equation of state and PengRobinson (P-R) equation of state is used to calculate it in this study, as expressed in Eq. (12).

$$
\begin{aligned}
p_{\text {EOS }}= & \frac{\rho R T}{1-b \rho} \\
& -\frac{a\left[1+\left(0.37464+1.54226 \omega-0.26992 \omega^{2}\right)\left(1-\sqrt{T / T_{c}}\right)\right]^{2} \rho^{2}}{1+2 b \rho-b^{2} \rho^{2}}
\end{aligned}
$$

where $a=0.45724 R^{2} T_{c}^{2} / p_{c}, b=0.0778 R T_{c} / p_{c}, T_{c}$ and $p_{c}$ are critical temperature and critical pressure, respectively.

\subsection{Liquid-vapor phase-change model}

Neglecting the viscous heat dissipation, the energy equation with phase-change source term $[10,26]$ is expressed as Eq. (13).

$\frac{\partial T}{\partial t}=-\mathbf{u} \cdot \nabla T+\frac{1}{\rho c_{v}} \nabla \cdot(\lambda \nabla T)-\frac{T}{\rho c_{v}}\left(\frac{\partial p_{\mathrm{EOS}}}{\partial T}\right)_{\rho} \nabla \cdot \mathbf{u}$

where the third term on the right-hand side is the phase-change source term [26], $c_{v}$ is the specific heat and $\lambda$ is the thermal conductivity. In this study, Eq. (13) is solved by finite-difference method. Define the right-hand side of Eq. (13) as $K(T)$, and the fourthorder Runge-Kutta scheme is adopted for time discretization.

$T(t+\Delta t)=T(t)+\frac{\Delta t}{6}\left(h_{1}+2 h_{2}+2 h_{3}+h_{4}\right)$

where $h_{1 \sim 4}$ can be expressed as follows:

$h_{1}=K(T(t)), h_{2}=K\left(T(t)+\frac{\Delta t}{2} h_{1}\right), h_{3}=K\left(T(t)+\frac{\Delta t}{2} h_{2}\right)$,

$h_{4}=K\left(T(t)+\Delta t h_{3}\right)$

\subsection{Electric field model for perfect dielectric}

According to the electrohydrodynamics theory [27], $\mathbf{F}_{\mathbf{e}}$ can be written as Eq. (16), where $q_{v}$ is the free charge density, $\varepsilon_{0}$ is the vacuum permittivity, $\varepsilon$ is the relative permittivity of fluid and $\mathbf{E}$ is the electric field intensity. On the right hand side of Eq. (16), the first term, second term and third term are Coulomb force, dielectric electrophoretic force and electrostriction force, respectively.

$\mathbf{F}_{\mathbf{e}}=q_{v} \mathbf{E}-\frac{1}{2} \mathbf{E} \cdot \mathbf{E} \nabla \varepsilon \varepsilon_{0}+\frac{\varepsilon_{0}}{2} \nabla\left(\rho \frac{\partial \varepsilon}{\partial \rho} \mathbf{E} \cdot \mathbf{E}\right)$

At present, many scholars have simulated many different EHD phenomena by LBM [28-33]. In this paper, it is assumed that the fluid is incompressible and perfect dielectric. There is no dynamic current in the fluid and the magneto-induced effect is neglected. Therefore, the Coulomb force and the electrostriction force can be neglected [31] and $\mathbf{F}_{\mathbf{e}}$ can be simplified into Eq. (17).

$\mathbf{F}_{\mathbf{e}}=-\frac{1}{2} \mathbf{E} \cdot \mathbf{E} \nabla \varepsilon \varepsilon_{0}$

Based on the above assumptions, the electric field vectors are irrotational and $\mathbf{E}$ can be solved [33] by $\nabla \cdot\left(\varepsilon \varepsilon_{0} \mathbf{E}\right)=0$. Since $\mathbf{E}$ is the gradient of electric potential $V$, i.e.E $=-\nabla V$, the distribution of electric potential can be expressed as Eq. (18).

$\nabla \cdot\left(\varepsilon \varepsilon_{0} \nabla V\right)=0$

In this study, Eq. (18) can be solved by the LB equation [28,31] as follows.

$\eta_{\alpha}\left(\mathbf{x}+\mathbf{e}_{\alpha} \Delta t, t+\Delta t\right)-\eta_{\alpha}(\mathbf{x}, t)=-\frac{1}{\tau_{s}}\left[\eta_{\alpha}(\mathbf{x}, t)-\eta_{\alpha}^{e q}(\mathbf{x}, t)\right]$

where $\tau_{\mathrm{s}}$ is the is the relaxation time and $\tau_{\mathrm{s}}=3 \varepsilon \varepsilon_{0}+0.5 . \eta_{\alpha}$ is the distribution function of electric potential, $\eta_{\alpha}{ }^{\mathrm{eq}}(\mathbf{x}, t)$ is its equilibrium distribution function.

$\eta_{\alpha}^{e q}(\mathbf{x}, t)=w_{\alpha} V$

The relationship between $\eta_{\alpha}$ and electric potential $V$ can be expressed as follows

$V=\sum_{\alpha} \eta_{\alpha}(\mathbf{x}, t)$ 
In this study, the periodic boundary condition is applied in $x$-direction and non-equilibrium extrapolation method is applied in $y$-direction. Concretely, the boundary conditions for the distribution functions are shown in Eqs. (22)-(25).

Left Boundary $(x=0)$ :

$\eta_{1}(0, y)=\eta_{1}\left(L_{x}, y\right), \eta_{5}(0, y)=\eta_{5}\left(L_{x}, y-1\right), \eta_{8}(0, y)=\eta_{8}\left(L_{x}, y+1\right)$

Right Boundary $\left(x=L_{x}\right)$ :

$\eta_{3}\left(L_{x}, y\right)=\eta_{3}(0, y), \eta_{6}\left(L_{x}, y\right)=\eta_{6}(0, y-1), \eta_{7}\left(L_{x}, y\right)=\eta_{7}(0, y+1)$

Top Boundary $\left(y=L_{y}\right)$ :

$\eta_{\alpha}\left(x, L_{y}\right)=\eta_{\alpha}^{e q}\left(x, L_{y}\right)+\eta_{\alpha}\left(x, L_{y}-1\right)-\eta_{\alpha}^{e q}\left(x, L_{y}-1\right), \alpha=0 \sim 8$

Bottom Boundary $(y=0)$ :

$\eta_{\alpha}(x, 0)=\eta_{\alpha}^{e q}(x, 0)+\eta_{\alpha}(x, 1)-\eta_{\alpha}^{e q}(x, 1), \alpha=0 \sim 8$

\section{Validation of numerical models}

\subsection{Validation of thermodynamic consistency}

Firstly, a flat interface problem is considered to compare the coexistence curves obtained by LBM and those given by Maxwell construction. In this simulation, a $100 \times 100$ lattice is adopted. The periodical boundary conditions are applied in $x$-direction and $y$-direction. Equation of state (12) is used, and the parameters in Eq. (12) are set as: $\omega=0.344, a=1 / 100, b=2 / 21, R=1$ and $T_{C}=0.017866$. The density field is initialized as $\rho(x, y)=\rho_{V}+0.5$ $\left(\rho_{L}-\rho_{V}\right)\left[\tanh \left(y_{1}\right)-\tanh \left(y_{2}\right)\right]$, where $y_{1}=2(y-25) / W, W=5$ is the initial phase interface thickness and $y_{2}=2(y-75) / W$.

Fig. 1 shows the comparison of the numerical coexistence curves with the coexistence curves given by Maxwell construction. As shown in Fig. 1, the results predicted by LBM calculations in this study agree well with those given by the Maxwell construction in both the liquid and vapor branches. Therefore, the rationality of the two-phase model used at present study could be proved.

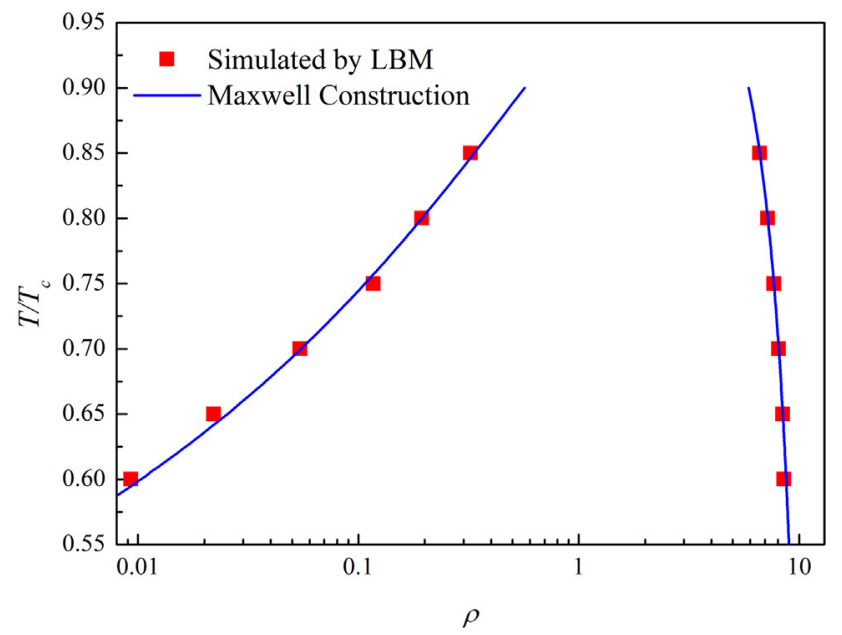

Fig. 1. Comparison of the numerical coexistence curves with the coexistence curves given by Maxwell construction.

\subsection{Validation of liquid-vapor phase-change model}

In order to validate the liquid-vapor phase-change model used at present study, the well-known D2 law is considered in this section. According to the D2 law, the time rate of change of the square of the evaporating droplet diameter is constant, i.e., $D^{2}(t) / D_{0}^{2}=1-k t$ [34]. The simulations are performed in a square domain $L_{x} \times L_{y}=100 \times 100$. The parameters in Eq. (12) are set as: $\omega=0.344, a=3 / 49, b=2 / 21, R=1$ and $T_{c}=0.109383$. Initially, a droplet with a radius of $R_{0}=20$ is located in the center of the computational domain. The temperature of the droplet is set to be its saturation temperature $T_{\text {sat }}\left(=0.86 T_{c}\right)$ and the temperature of the surrounding vapor is given by $T_{g}=T_{\text {sat }}+\Delta T$. The kinematic viscosity is taken as $v=0.1$ in the whole computational domain and the thermal conductivity is chosen to be constant: $\lambda=0.3$. The specific heat at constant volume is set to $c_{v}=3.29$. At the boundaries, a constant temperature $\left(T_{g}\right)$ is applied.

Two cases are simulated: Case A with $\Delta T=0.14 T_{c}$ and Case B with $\Delta T=0.28 T_{c}$. Jacob numbers for Case $A$ and Case $B$ are $J a=0.1$ and $J a=0.2$, respectively, where $J a=c_{v}\left(T_{w}-T_{s a t}\right) / h_{f g}$ and $h_{f g}$ is the latent heat of vaporization. Fig. 2 represents the droplet evaporation processes simulated by LBM in this paper, with the comparison with Sadeghi et al.'s 3D numerical results [12]. As shown in Fig. 2, in both cases, the value of $\left(D / D_{0}\right)^{2}$ decreases linearly over time. Quantitatively, the parameter $k$ in the law of $D^{2}(t) / D_{0}^{2}=1-k t$ is given by $6 \times 10^{-6}$ and $1 \times 10^{-5}$ for Case $A$ and Case B, respectively. Obviously, the parameter $k$ of Case B is about two times that of Case $A$. In addition, the numerical results simulated by LBM in this paper agree well with those given by Sadeghi et al. [12]. Therefore, the rationality of our two-dimensional (2D) liquid-vapor phase-change model can be proved.

\subsection{Validation of electric field model}

The deformation of a perfect dielectric droplet in a uniform electric field are simulated to validate the electric field model used at present study. It should be noted that many scholars studied the electrohydrodynamic drop deformation with the leaky dielectric theory $[29,35]$. Since their electric field models are quite different from ours, their numerical results can't be used to validate the electric field model for perfect dielectric used at present study.

In this simulation, a rectangular computational domain with a size of $8 R_{d} \times 8 R_{d}$ is used, and a circular droplet with the radius of $R_{d}=25$ is located at the center of the computational domain. The boundary conditions are period and Neumann boundary

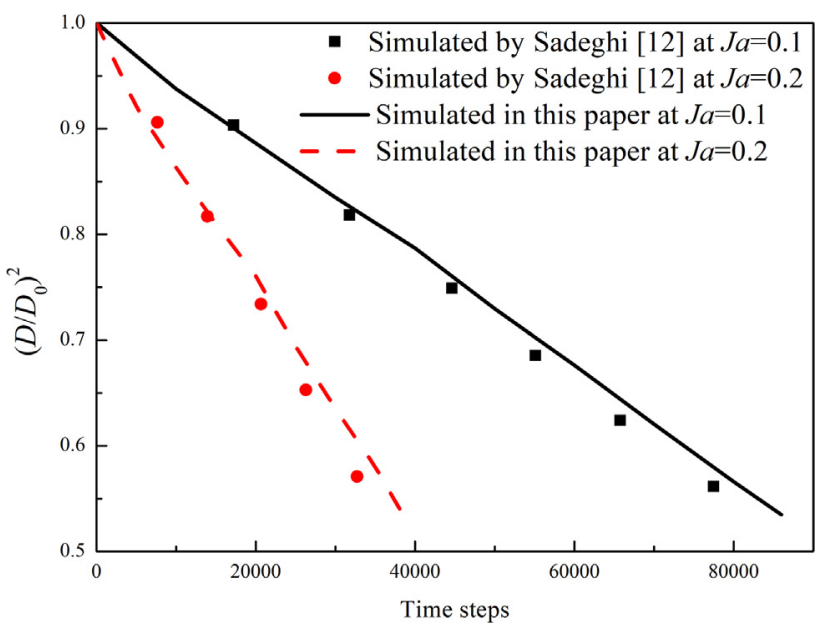

Fig. 2. Variation of the square of the non-dimensional droplet diameter with time 
conditions in $x$-direction and $y$-direction, respectively, thus a uniform electric field is imposed in the vertical direction. The permittivity ratio of vapor to liquid is $\varepsilon_{V} / \varepsilon_{L}=0.2$. According to Sherwood's theoretical study [36], when $\varepsilon_{V} / \varepsilon_{L}$ is kept constant, the droplet deformation can be fully decided by Eq. (26).

$\mathrm{Bo}_{e}^{*}=\frac{\varepsilon_{0} \varepsilon_{L} E_{0}^{2} R_{d}}{\gamma}$

where $E_{0}$ is the characteristic electric intensity.

Fig. 3 represents the numerical results of deformation rate, i.e. $D_{F}$, under different value of $\mathrm{Bo}_{e}{ }^{*}$, with the comparison with the theoretical results [36] and the numerical results of other scholars [37-39] using various methods. It can be seen from Fig. 3 that the numerical results obtained in this study are consistent with the theoretical result [36] and the numerical results by other scholars [37-39]. Therefore, the rationality of our electric field model can be proved.

\subsection{Convergence study}

The simulation results of film boiling with three different meshes (including $430 \times 100,650 \times 150$ and $910 \times 210$ cells) are compared in terms of Nusselt number. These meshes correspond to Case A, Case B, and Case C, respectively. The parameters in Eqs. (6) and (12) set as: $\sigma=1.2, \omega=0.344, a=3 / 49, b=2 / 21$, $R=1$ and $T_{c}=0.1094$. The initial setting of the computational domain is a liquid $\left(0 \leq y \leq 0.6 L_{y}\right)$ below its vapor $\left(0.6 L_{y}<y \leq L_{y}\right)$, and the temperature in the domain is the corresponding coexistence temperature $T_{s a t}=0.86 T_{c}$. The bottom wall is a heated surface with a high temperature $T_{w}$. In the simulations, $\rho_{L}=6.5, \rho_{V}=0.38$, $c_{v}=1.76, v_{L}=0.1, v_{V}=1.27, g_{y}=0.00003, T_{w}=1.828 T_{c}, G r=16.97$ and $J a=0.371$.

Fig. 4 represents the numerical results of the evolution of Nusselt number for different unit cell sizes. As shown in Fig. 4, the numerical results of Case $B$ and Case $C$ are very close with each other. However, there is a significant deviation between the simulation results of Case A and those of the other two cases. Therefore, considering the trade-off between the accuracy and economy, the second grid resolution (Case B) is adopted to study the pool boiling heat transfer. In addition, after the film boiling processes are stable, the time-averaged Nusselt numbers of cases A, B and C are 1.85, 1.62 and 1.61 , respectively. The $3 \mathrm{D}$ numerical result of the timeaveraged Nusselt number by Sadeghi et al. [13] under the same conditions is found to be 1.33 . Therefore, compare with the results by Sadeghi et al. [13], the deviations of the time-averaged Nusselt numbers at cases A, B and C are $39.1 \%, 21.8 \%$ and $21.1 \%$, respec-

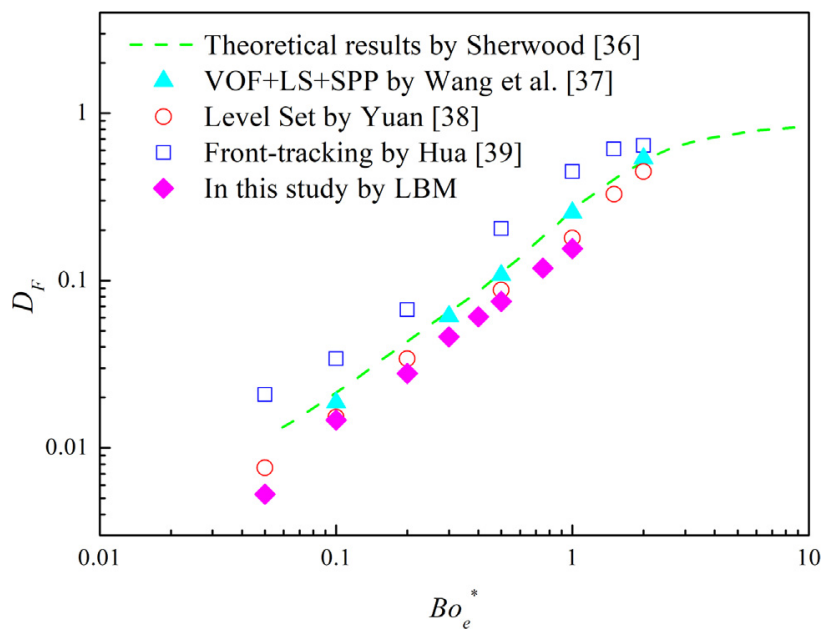

Fig. 3. Droplet deformation rates $\left(D_{F}\right)$ with different value of $B o_{e}{ }^{*}$.

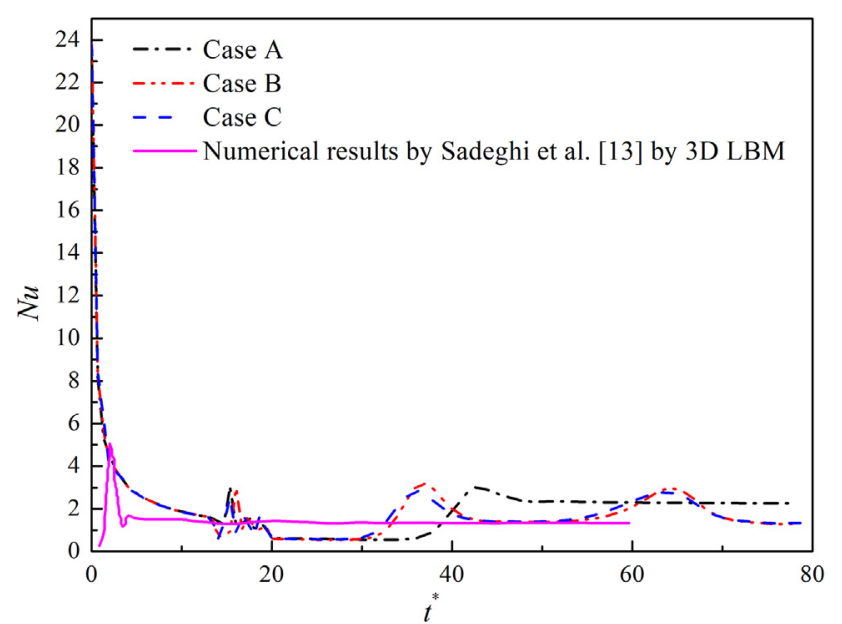

Fig. 4. Convergence study of Nusselt number for different unit cell sizes as a function of time for three cases, with the comparison with the numerical results of Sadeghi et al. [13] by 3D LBM.

tively. It's undeniable that there are still some deviations between the $2 \mathrm{D}$ simulation results and the 3D simulation results [13]. Therefore, it's necessary to investigate the effect of EHD on pool boiling heat transfer by 3D simulations in further study.

\section{Numerical results}

\subsection{Physical model and calculation parameters}

As shown in Fig. 5, the pool boiling heat transfer in presence of a uniform electric field is simulated in the computational domain with $L_{x} \times L_{y}$, where $L_{x}=650, L_{y}=150$. A heated surface with a thickness of $H_{s}=10$ is located at the bottom of computational domain and the outer wall temperature of heated surface $T_{w}$ is higher than the saturation temperature of fluid $T_{s a t}\left(=0.86 T_{c}\right)$. The periodic boundary condition is applied in the $x$-direction, the convective boundary condition is applied at the top boundary. Meanwhile, the heated surface is set as high electrode with the electric potential of $V_{1}$. A low electrode is located at the top boundary and is linked with the ground (i.e., $V_{2}=0$ ). In this chapter, the characteristic electric field intensity $E_{0}$ can be expressed as $E_{0}=\left(V_{1}-V_{2}\right)$ / $\left(L_{y}-H_{s}\right)$. Initially, the computational domain is filled with saturation liquid.

The parameters in Eqs. (6) and (12) set as: $\sigma=1.2, \omega=0.344$, $a=3 / 49, b=2 / 21, R=1, T_{c}=0.109383$ and $p_{c}=0.089355$. The kinematic viscosity of liquid and vapor are $v_{L}=0.1$ and $v_{V}=0.5 / 3$, respectively. The specific heat $c_{v}=6$, thermal conductivity $\lambda=\rho c_{v} \chi$, where $\chi=0.028 / c_{v}$. The thermal conductive ratio of liquid to vapor is $\lambda_{L} / \lambda_{V}=\rho_{L} / \rho_{V}=17$. The thermal conductivity of heated surface $\lambda_{S}=10 \lambda_{L}$. The relaxation times in Eq. (2) set as: $\tau_{\rho}=1.0$, $\tau_{e}=1.25, \tau_{\varsigma}=1.25, \tau_{j}=1.0, \tau_{q}=1 / 1.1$ and $\tau_{v}=3 v+0.5$, where $v=\frac{v_{L}\left(\rho-\rho_{V}\right)+v_{V}\left(\rho_{L}-\rho\right)}{\rho_{L}-\rho_{V}}$. In Eq. (9), the coefficient $G_{w}$ equals 0 , thus the contact angle is $44.5^{\circ}$. The surface tension $\gamma$ is found to be 0.27 according to Laplace equation of capillary (detailed explanation is shown in Appendix). The gravitation acceleration is set as $g_{y}=3 \times 10^{-5}$. Using the method proposed by Gong and Cheng [40], the latent heat of vaporization $h_{f g}$ is found to be 0.5032 . The permittivity of vacuum is $\varepsilon_{0}=2.236$ and the permittivity ratio of vapor to liquid is $\varepsilon_{V} / \varepsilon_{L}=0.4472$.

$q(t)$ is defined as the space-averaged heat flux on heated surface, $q_{w}$ is defined as the time- and space-averaged heat flux on heated surface. 


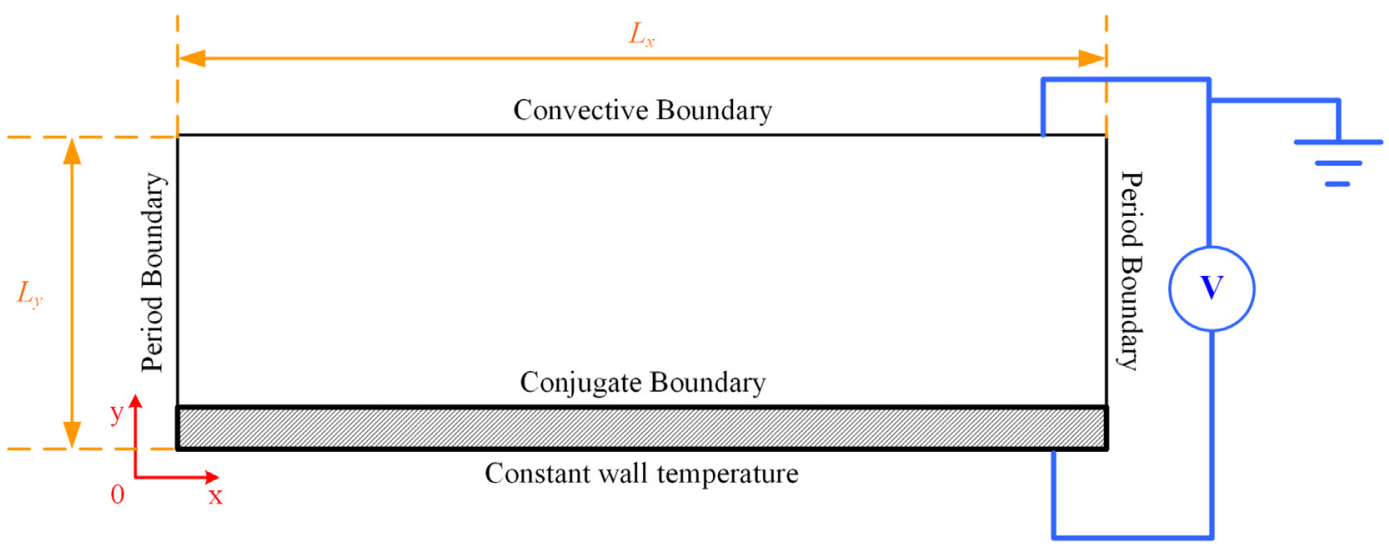

Fig. 5. Physical model to study the influence of a uniform electric field on pool boiling.

$q(t)=-\frac{\lambda_{s}}{L_{x}} \int_{0}^{L_{x}} \frac{\partial T(x, 0)}{\partial y} d x, q_{w}=\frac{\int_{t_{1}}^{t_{2}} q(t) d t}{t_{2}-t_{1}}$

where $t_{2}$ and $t_{1}$ are two different moments after the boiling process is stable.

\subsection{Pool boiling in the absence of an electric field}

Fig. 6 represents the pool boiling processes under different outer wall temperatures in the absence of an electric field (i.e., $E_{0}=0$ ). Since all of the information in the computational domain is symmetrical along $x=L_{x} / 2$, only the snapshots in the range of $x \leq L_{x} / 2$ are displayed. As shown in Fig. 6(a), at $T_{w}=0.11$, there are only a small number of bubbles appears at the heated surface and the interaction between vapor bubbles is weak. Obviously, the boiling at $T_{w}=0.11$ is in the partial nucleate boiling regime. As shown in Fig. $6(\mathrm{~b})$, at $T_{w}=0.13$, the number of vapor bubbles increases obviously and the size of vapor bubbles becomes larger compared with Fig. 6(a). What's more, the interaction between the vapor bubbles is stronger and the boiling process is more intense than that in Fig. 6(a), as well. Therefore, it's reasonable to believe the boiling at $T_{w}=0.13$ is in the fully developed nucleate boiling regime. As shown in Fig. $6(\mathrm{c})$, at $T_{w}=0.14$, a great portion of the heated surface is covered by vapor patches. Since vapor is less capable of conducting heat, these vapor patches will essentially insulate the liquid from the heated surface. Therefore, the boiling at $T_{w}=0.14$ is in transition boiling regime. As shown in Fig. 6(d), at $T_{w}=0.16$, the heated surface is covered with a
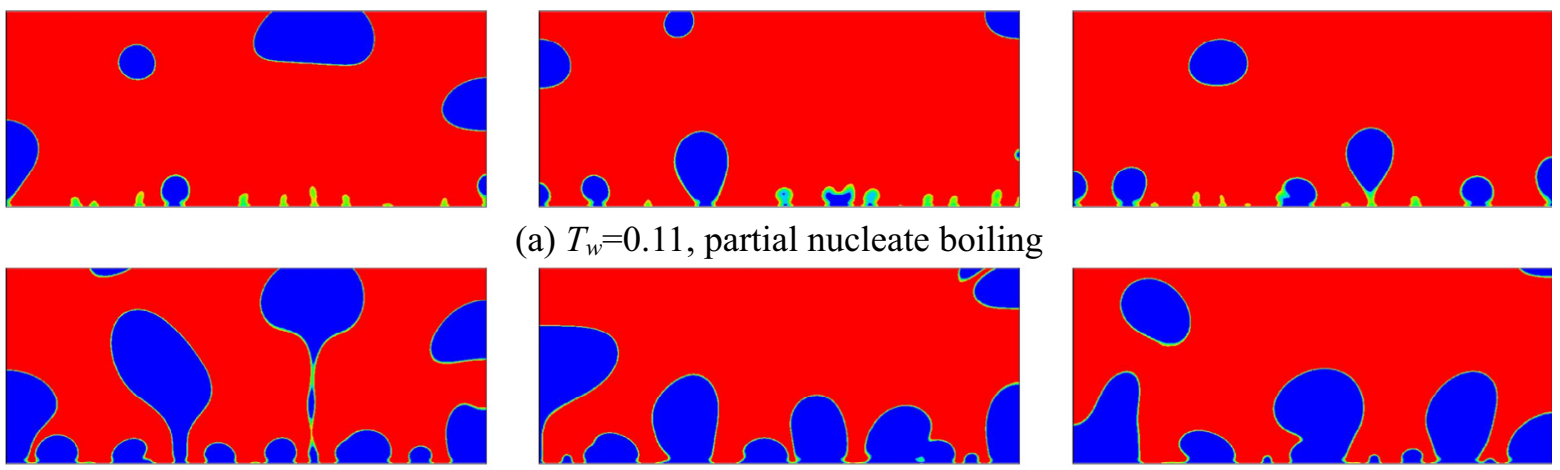

(a) $T_{w}=0.11$, partial nucleate boiling
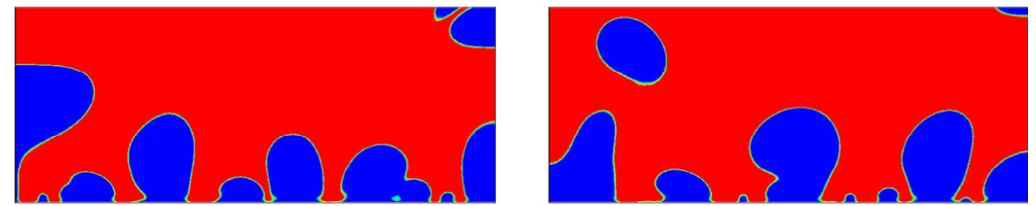

(b) $T_{w}=0.13$, fully developed nucleate boiling
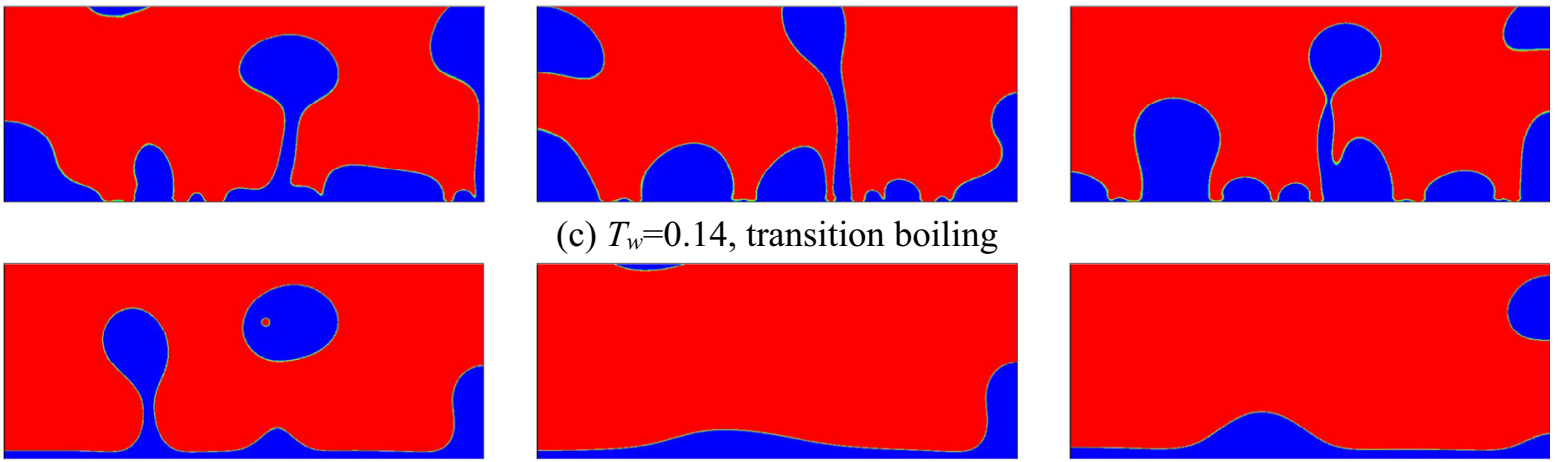

(c) $T_{w}=0.14$, transition boiling
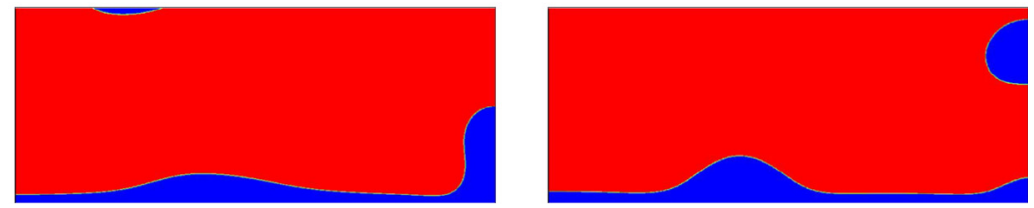

(d) $T_{w}=0.16$, film boiling

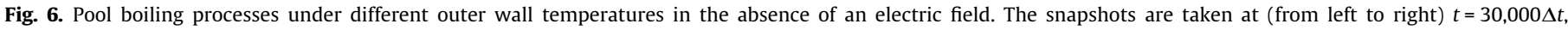
$t=36,000 \Delta t$ and $t=42,000 \Delta t$. 


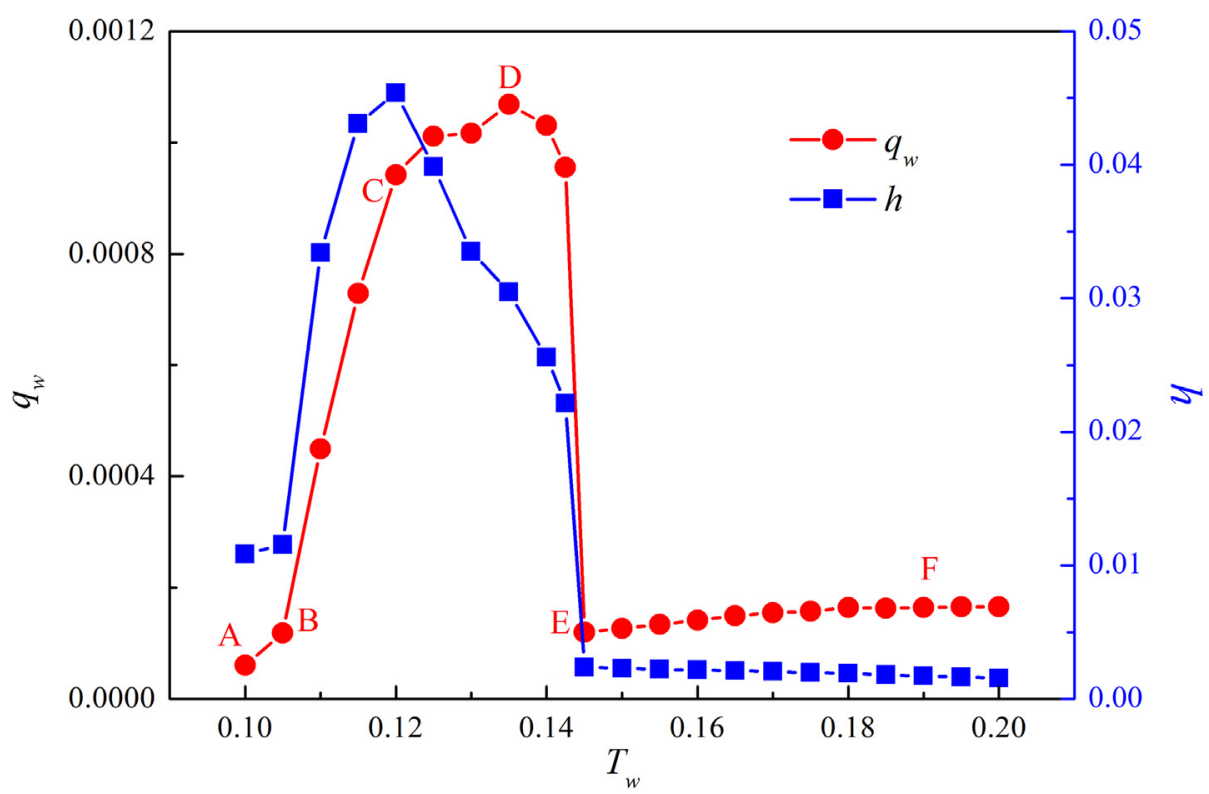

Fig. 7. The influence of outer wall temperature $T_{w}$ on space- and time-averaged heat flux $\left(q_{w}\right)$ and heat transfer coefficient $(h)$ in the absence of an electric field.

continuous vapor film, which completely insulates the liquid from the heated surface. Obviously, the boiling at $T_{w}=0.16$ is in film boiling regime.

Fig. 7 displays the influence of outer wall temperature, i.e., $T_{w}$, on the time- and space-averaged heat flux $q_{w}$ and heat transfer coefficient $h$, where $h=q_{w} /\left(T_{b}-T_{\text {sat }}\right)$ and $T_{b}$ is the inner wall temperature. In Fig. 7, the curve $\mathrm{AB}$ (i.e., $T_{w} \leq 0.105$ ) represents the natural convection regime. At this case, the wall superheat is insufficient to support bubble formation and growth. Point " $\mathrm{B}$ " is the onset of nucleate boiling (ONB). The curve BC (i.e., $0.105 \leq T_{w} \leq 0.120$ ) represents the partial nucleate boiling regime. In this regime, increasing $T_{w}$ could enhance both $q_{w}$ and $h$. The curve CD (i.e., $0.120 \leq T_{w} \leq 0.135$ ) represents the fully developed nucleate boiling regime. In this regime, with an increase in $T_{w}, q_{w}$ increases, while $h$ decreases gradually. At point " $D$ ", $q_{w}$ reaches its maximum value, thus it can be found that CHF equals to 0.00107 . The curve $\mathrm{DE}$ (i.e., $0.135 \leq T_{w} \leq 0.145$ ) represents the transition boiling regime, in which increasing $T_{w}$ would lead to a decrease in both
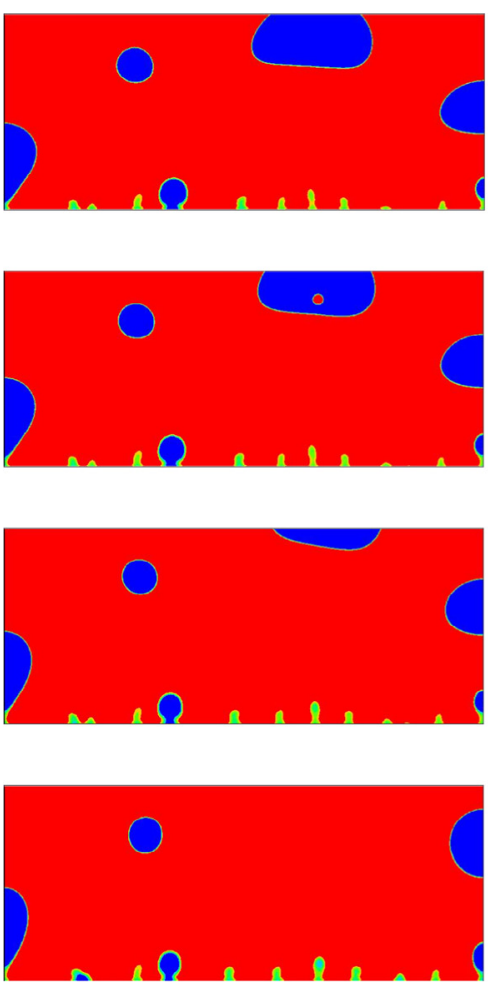

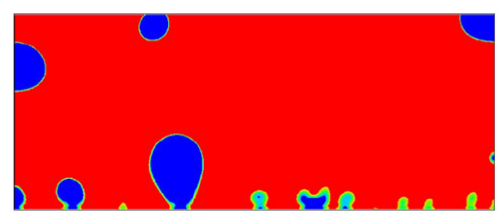

(a) $E_{0}=0$

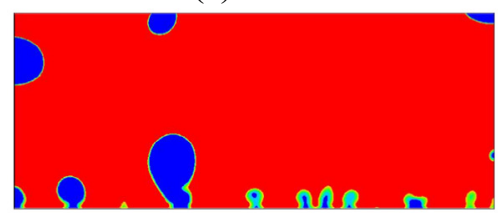

(b) $E_{0}=0.0286$

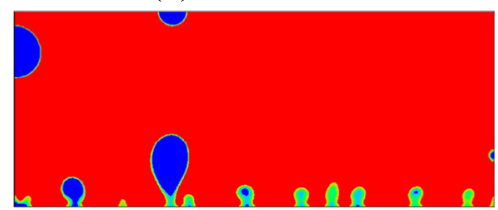

(c) $E_{0}=0.0571$

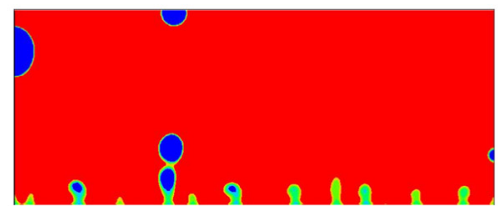

(d) $E_{0}=0.0857$
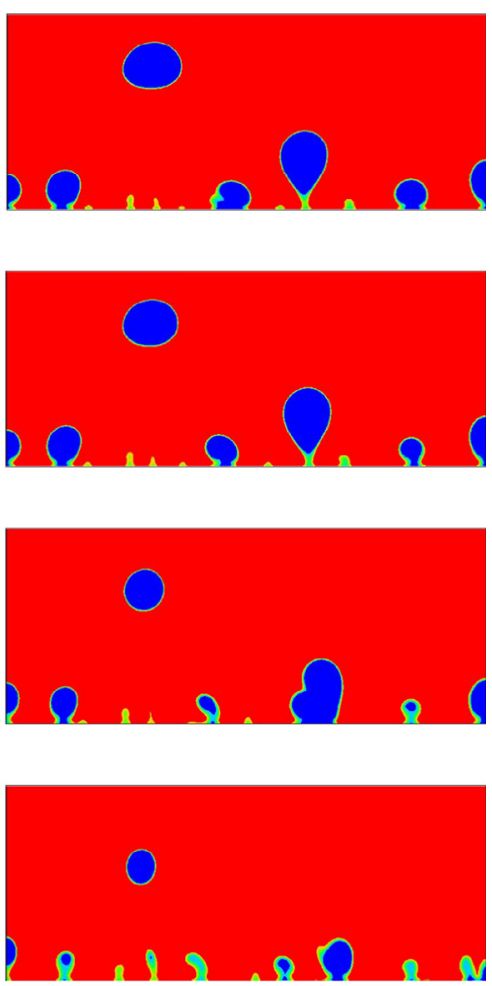

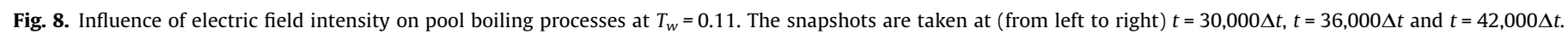


$q_{w}$ and $h$. At point "E", $q_{w}$ reaches its minimum value, thus it can be found that minimum film boiling heat flux (MHF) is 0.00012 . The curve $\mathrm{EF}$ (i.e., $T_{w} \geq 0.145$ ) represents the film boiling regime. With an increase in $T_{w}, q_{w}$ increases slowly while $h$ decreases slightly.

\subsection{The effect of a uniform electric field on pool nucleate boiling progresses}

Fig. 8 represents the pool boiling under different conditions of electric field intensity $\left(E_{0}=0,0.0286,0.0571\right.$ and 0.0857$)$ at $T_{w}=0.11$. As shown in Fig. 8, the effect of the uniform electric field on bubble dynamics during nucleate boiling is limited at $T_{w}=0.11$. For all cases in Fig. 8, the vapor bubbles constantly nucleate on, grow on and depart away from the heated surface. The number of bubbles in the computational domain is small and the interaction between vapor bubbles is weak.

Fig. 9 displays the pool boiling under different electric field intensities (i.e., $E_{0}$ ) at $T_{w}=0.13$. Compared with Fig. 8, the active site density increases and the interaction between bubbles becomes stronger when $T_{w}$ is increased into 0.13 . Obviously, all of the boiling processes in Fig. 9 are in the fully developed nucleate boiling regime. As displayed in Fig. 9, increasing $E_{0}$ could make the bubbles become slenderer in shape. Fig. 10 gives the evolution of the area share of vapor on the heated surface, i.e. $Z_{V}$, under different electric field intensities at $T_{w}=0.13$, where $Z_{V}=A_{V} /\left(A_{V}+A_{L}\right), A_{V}$ is the contact area between heated surface and vapor and $A_{L}$ is the contact area between heated surface and liquid. It can be concluded from Fig. 10 that increasing $E_{0}$ could decrease the contact area between heated surface and vapor. Since vapor is less capable of conducting heat, the boiling heat transfer performance will be enhanced under the effect of a uniform electric field at $T_{w}=0.13$.

Fig. 11 represents the effect of electric field intensity on timeand space-averaged heat flux $q_{w}$ under different outer wall temperatures during nucleate boiling, where $r_{q}$ represents the enhanced

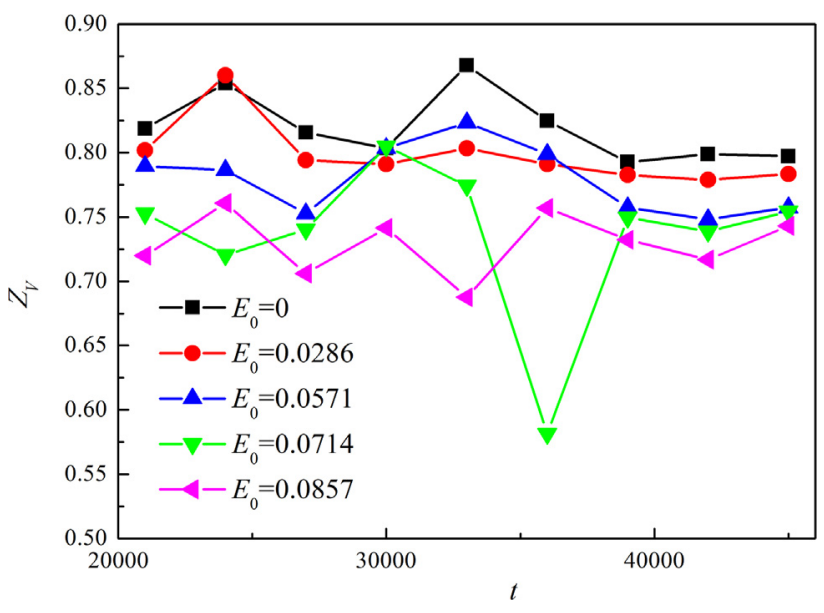

Fig. 10. Influence of electric field intensity on the evolution of the contact area between heated surface and vapor, i.e. $Z_{V}$, at $T_{w}=0.13$.

ratio of $q_{w}$ due to the electric field. As shown in Fig. 11, at $T_{w}=0.11, r_{q}$ almost stays constantly at 1 under different electric field intensities. This result means that a uniform electric field has little effect on boiling heat transfer during nucleate boiling at a low wall superheat. It's because the electric field intensity has little influence on bubble dynamics at this time, as displayed in Fig. 8. In addition, at $T_{w}=0.12, T_{w}=0.125$ and $T_{w}=0.13$, increasing electric field intensity could enhance the value of $r_{q}$ gradually. The reason has been analyzed in Fig. 9 and Fig. 10. It can also be found in Fig. 11 that increasing outer wall temperature could enhance the value of $r_{q}$ gradually at a given electric field intensity $\left(E_{0} \neq 0\right)$. Therefore, in the nucleate boiling regime, the enhanced effect of a uniform electric field on boiling heat transfer becomes stronger with an increase in $T_{w}$.
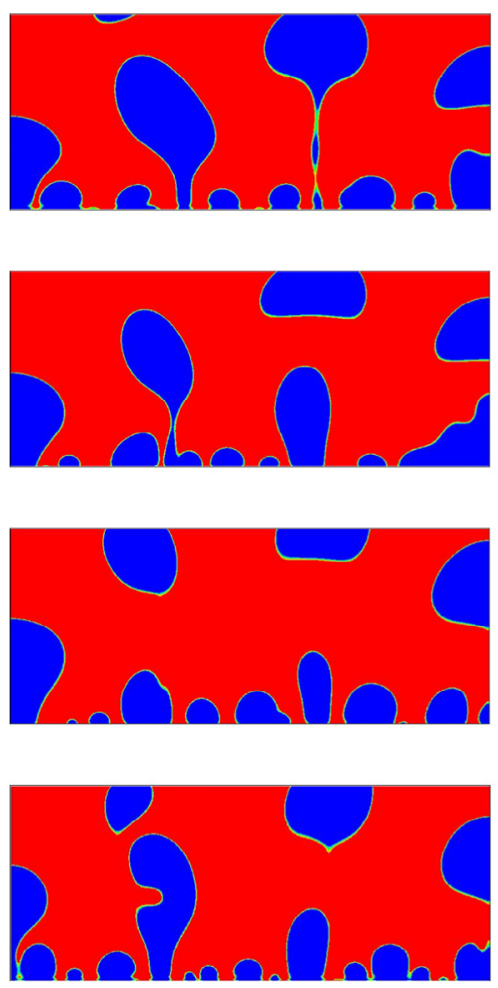

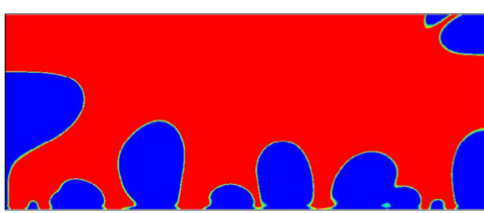

(a) $E_{0}=0$

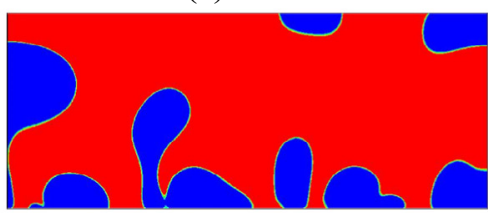

(b) $E_{0}=0.0286$

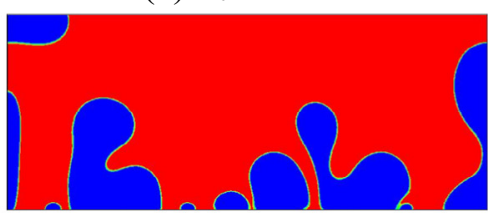

(c) $E_{0}=0.0571$

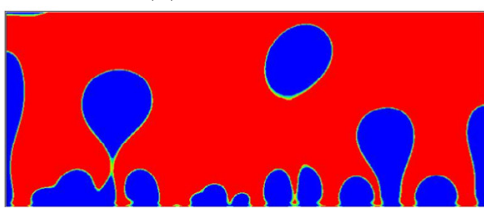

(d) $E_{0}=0.0857$
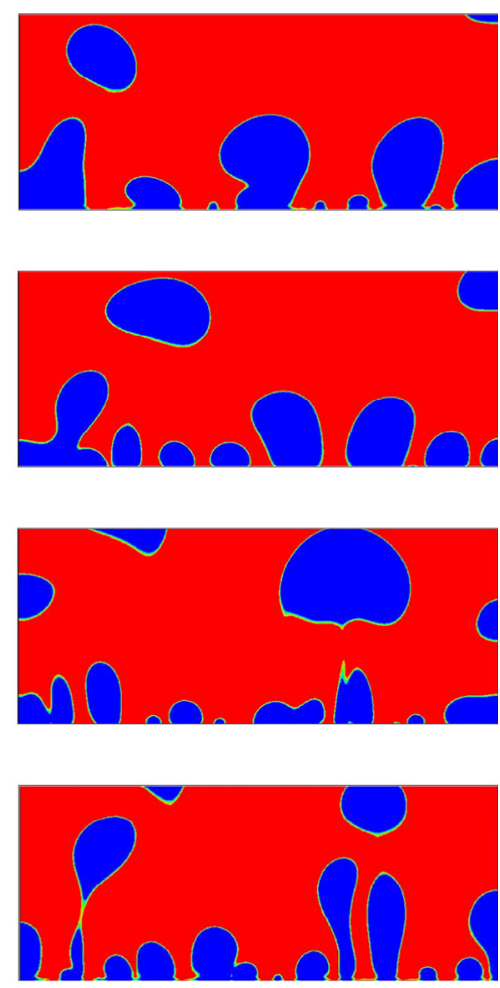

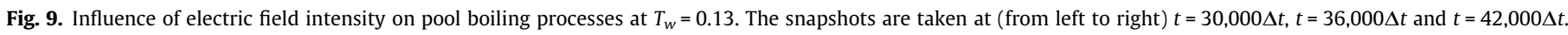




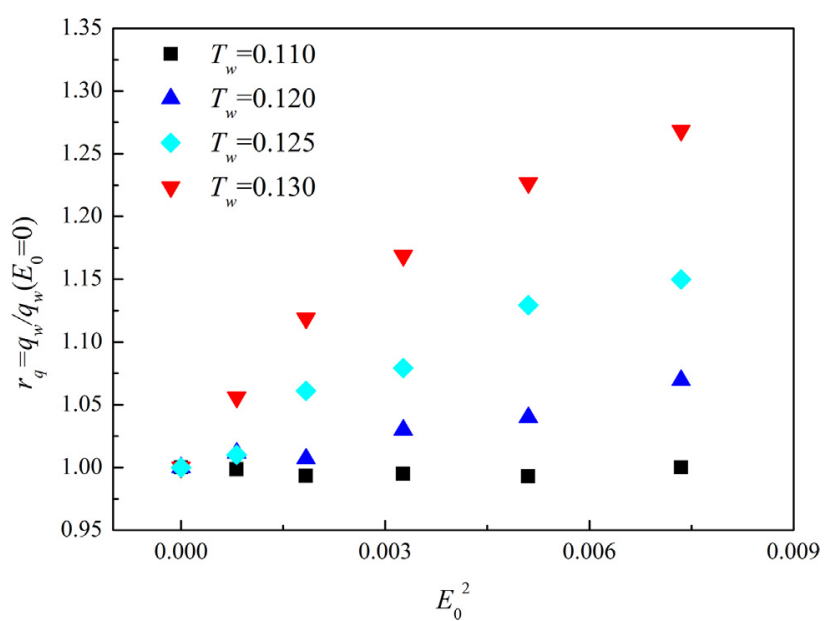

Fig. 11. Variation of time- and space-averaged heat flux, i.e. $q_{w}$, with square of electric field intensity under different outer wall temperatures during nucleate boiling.

\subsection{The effect of a uniform electric field on film boiling progresses}

Fig. 12 represents the pool boiling processes under different electric field intensities (i.e., $E_{0}$ ) at $T_{w}=0.20$. As shown in Fig. 12 (a), due to the high wall superheat, the heated surface is covered by vapor film, which completely insulates the liquid from the heated surface. Therefore, the boiling heat transfer performance is very low at this case. As shown in Fig. 12(b), the boiling at $E_{0}=0.0286$ is still in the film boiling regime. Compared with Fig. 12(a), the active site density is unchanged, while bubble release frequency increases slightly. As shown in Fig. 12(c), the boiling at $E_{0}=0.0429$ is still in film boiling regime. However, under the effect of a uniform electric field, active site density and bubble release frequency increase obviously. This result agrees well with the numerical results by CLSVOF method in Ref. [41]. As shown in Fig. 12(d), the boiling at $E_{0}=0.0714$ is in the transition boiling regime because a great portion of the heated surface is covered by vapor patches. The thermal conduction between heated surface and liquid isn't obstructed completely, so the boiling heat transfer at $E_{0}=0.0714$ is much greater than other cases in Fig. 12 . It can be concluded from Fig. 12 that bubble dynamics during film boiling could be affected by a uniform electric field, boiling heat transfer performance could be enhanced and even the boiling regime could be changed when $E_{0}$ is large enough.

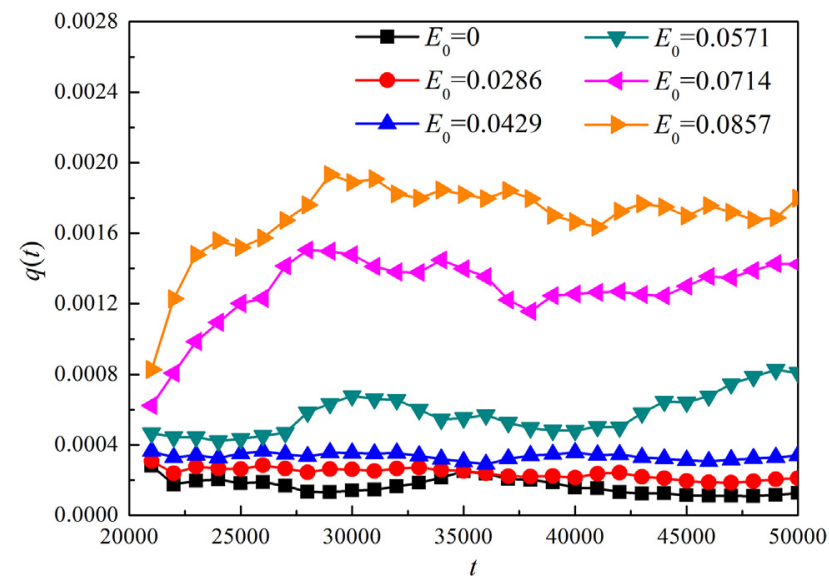

Fig. 13. The evolution of space-averaged heat flux, i.e. $q(t)$, during film boiling under different electric field intensities at $\Delta T=0.20$.
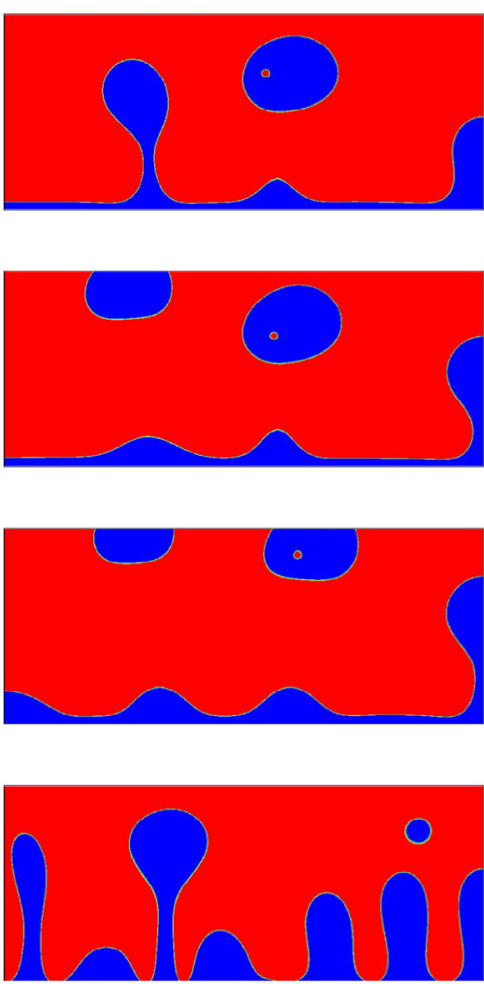

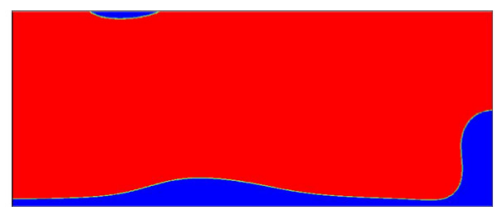

(a) $E_{0}=0$

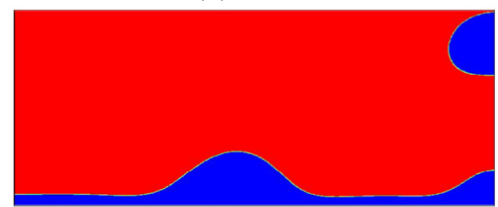

(b) $E_{0}=0.0286$

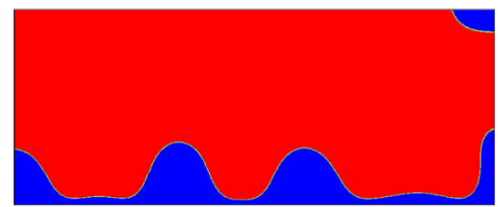

(c) $E_{0}=0.0429$

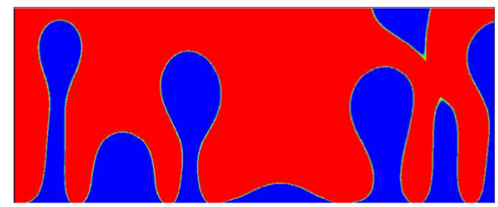

(d) $E_{0}=0.0714$
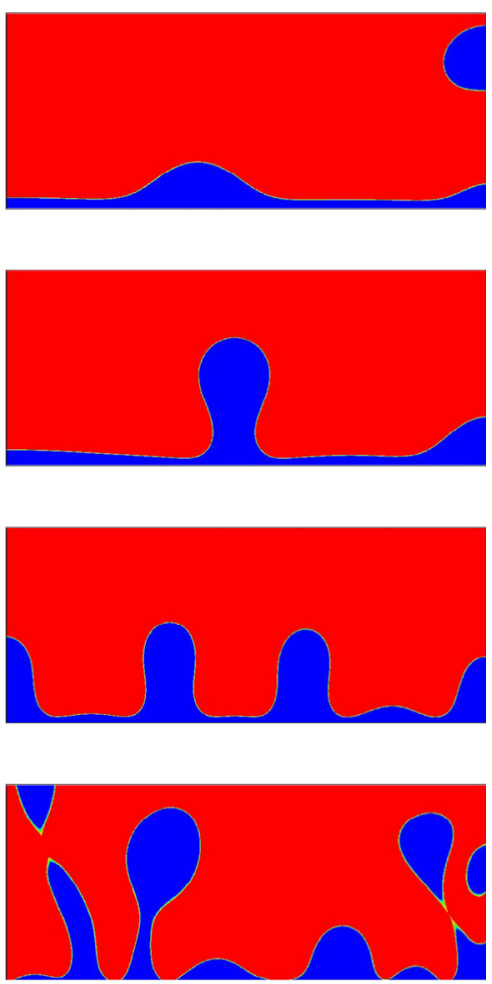

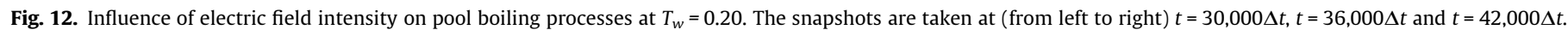


Fig. 13 represents the influence of electric field intensity on the evolution of $q(t)$ at $T_{w}=0.20$. As shown in Fig. 13, when $E_{0} \leq 0.0429, q(t)$ is small and stable, and $q(t)$ increases slightly with an increase in electric field intensity. As shown in Fig. 12(a)-(c), when $T_{w}=0.20$ and $E_{0} \leq 0.0429$, the boiling is in film boiling regime, the heated surface is covered with a continuous vapor film. Therefore, $q(t)$ is small and stable. In presence of a uniform electric field, the active site density and bubble release frequency increase, thus $q(t)$ increases slightly with an increase in electric field intensity when $E_{0} \leq 0.0429$. Moreover, as shown in Fig. 13, when $E_{0} \geq 0.0571, q(t)$ fluctuates significantly and increases obviously with an increase in $E_{0}$. As shown in Fig. 12(d), when $E_{0}$ is large enough, the boiling is in transition boiling regime instead of film boiling regime, thus $q(t)$ increases obviously. In addition, the transition boiling can be viewed as an unstable combination of the nucleate boiling and the film boiling [10], thus $q(t)$ fluctuates significantly.

\subsection{The influence of electric field intensity on boiling curves}

Fig. 14 represents the boiling curves under different electric field intensities (i.e., $E_{0}$ ). As shown in Fig. 14, when wall temperature is low (i.e., $T_{w} \leq 0.12$ ), electric field intensity has little effect on the time- and space-averaged heat flux $q_{w}$. According to the analysis in Figs. 9 and 10, when wall superheat is higher but $q_{w}$ doesn't reach $\mathrm{CHF}$, the boiling is in fully developed nucleate boiling regime. At this case, the uniform electric field has a great impact on the boiling curves. At given a wall temperature, increasing $E_{0}$ would raise $q_{w}$ obviously. CHF and the outer wall temperature at $\mathrm{CHF}$ point increase gradually with an increase in $E_{0}$. For example, compared with the case at $E_{0}=0$, when $E_{0}=0.0857$, CHF is enhanced from 0.00107 to 0.00174 , and the outer wall temperature at $\mathrm{CHF}$ point is raised from 0.135 to 0.190 , indicating that increasing electric field intensity could enhance the upper limit of heat load for safe operation of equipment. In addition, increasing electric field intensity could raise both MHF and the outer wall temperature at MHF point.

Since CHF is of great importance in engineering, some correlations have been proposed to predict the influence of electric field intensity on CHF. The correlations of Berghmans [42] and Johnson [43] are the most widely used.

$$
\begin{aligned}
& r_{\mathrm{CHF}}=q_{w, \mathrm{CHF}} / q_{w, \mathrm{CHF}}\left(E_{0}=0\right)=\left(E l^{*}+\left(E l^{* 2}+1\right)^{1 / 2}\right)^{1 / 2}, \\
& \mathrm{El}^{*}=\frac{B E_{0}^{2}}{\sqrt{3\left(\rho_{L}-\rho_{V}\right) g_{y} \gamma}}
\end{aligned}
$$

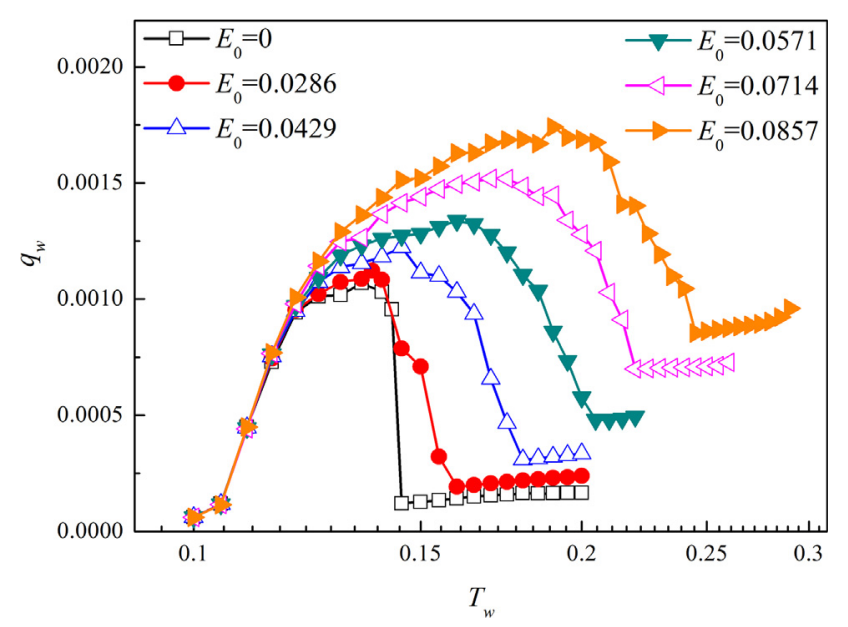

Fig. 14. Influence of electric field intensity on boiling curves.

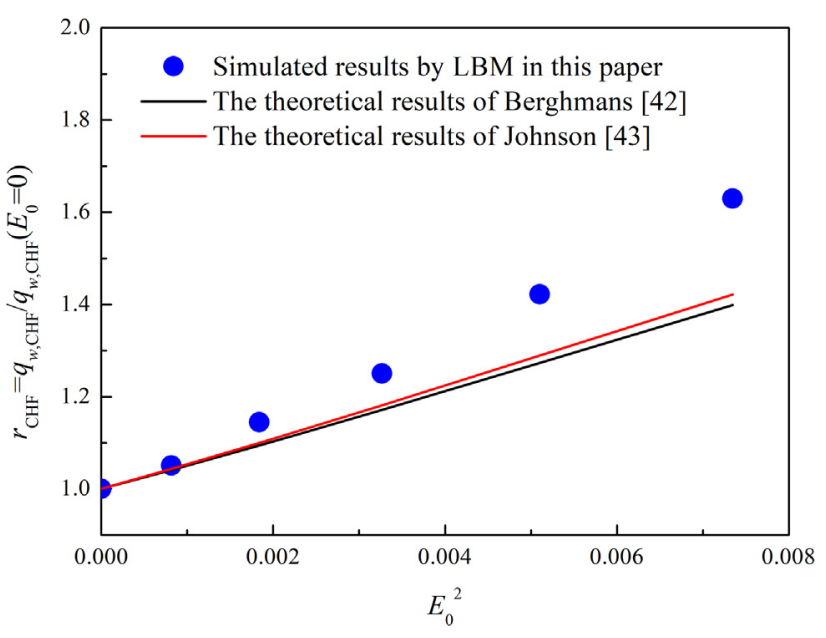

Fig. 15. Influence of electric field intensity on $\mathrm{CHF}$, with the comparison with the theoretical results of Berghmans [42] and Johnson [43].

where $r_{\mathrm{CHF}}$ is the enhancement ratio of CHF under a uniform electric field, $q_{w, \mathrm{CHF}}\left(E_{0}=0\right)$ represents the critical heat flux when $E_{0}=0$. In Berghmans's correlation [42], $B=\varepsilon_{0} \varepsilon_{V}$. While in Johnson's correlation [43], $B=\frac{\varepsilon_{0} \varepsilon_{L}\left(\varepsilon_{L}-\varepsilon_{V}\right)^{2}}{\varepsilon_{V}\left(\varepsilon_{L}+\varepsilon_{V}\right)}$.

Fig. 15 displays the influence of square of electric field intensity, i.e. $E_{0}^{2}$, on $r_{\mathrm{CHF}}$ with the comparison with the theoretical results calculated by Berghmans's correlation [42] and Johnson's correlation [43]. As shown in Fig. 15, $r_{\mathrm{CHF}}$ increases linearly with an increase in $E_{0}^{2}$. When $E_{0}=0.0857, r_{\mathrm{CHF}}=1.63, \mathrm{CHF}$ has been enhanced by $63 \%$. The deviations between the numerical results in this paper and the theoretical results of Berghmans [42] and Johnson [43] are $16.6 \%$ and $14.8 \%$, respectively. It can be concluded that our numerical results agree well with the theoretical results of Berghmans [42] and Johnson [43], and our numerical model is capable of simulating the pool boiling heat transfer under a uniform electric field.

\section{Concluding remarks}

In this paper, the two-dimensional lattice Boltzmann model was developed to simulate the pool boiling in presence of an external electric field. In this model, the pseudopotential model was coupled with phase-change model and electric field model. The pool boiling process in presence of a uniform electric field is simulated. The influence of electric field intensity on averaged heat flux on the heated surface under different wall superheats was analyzed, and the influence of electric field intensity on boiling curves and critical heat flux was also investigated. It can be concluded that:

(1) In the partial nucleate boiling regime, a uniform electric field has limited influence on bubble dynamics and time- and space-averaged heat flux $q_{w}$ on the heated surface.

(2) In the fully developed nucleate boiling regime, increasing electric field intensity could raise $q_{w}$. In addition, the effect of electric field intensity on $q_{w}$ becomes greater with an increase in wall superheat.

(3) In the film boiling regime, when electric field intensity is small, increasing electric field intensity would enhance $q_{w}$ increases slightly. However, after the electric field intensity reaches a certain value, $q_{w}$ increases rapidly because the film boiling is converted into transition boiling.

(4) Increasing electric field intensity could expand the nucleate boiling region, raise both critical heat flux and the wall temperature at critical heat flux point. MHF and the wall temperature at MHF point increase with an increase in electric field intensity, as well. 


\section{Conflict of interest statement}

We declare that we have no financial or personal relationships with other people or organizations that can inappropriately influence our work, there is no conflict of interest in the manuscript entitled "Numerical study on saturated pool boiling heat transfer in presence of a uniform electric field using lattice Boltzmann method".

\section{Acknowledgement}

This work is supported financially by the joint fund between the Chinese Academy of Sciences (CAS) and National Natural Science Foundation of China (NSFC) under the Grant of U1738105.

\section{Appendix A. Determination of the surface tension and contact angle}

According to Laplace's law, the pressure difference $\Delta P$ across a circular interface is related to the surface tension $\gamma$ and the droplet radius $R_{d}$ via Eq. (A-1). In order to obtain the surface tension, the pressure difference between inside and outside of saturated droplets of different radii are calculated using the two-phase flow model in Section 2.1. A $200 \times 200$ lattice is adopted, the buoyancy force is ignored and the temperature of the whole computational domain is set to be the saturated temperature of fluid, i.e., $T_{\text {sat }}=0.86 T_{c}$. The simulation results of $\Delta \boldsymbol{P}$ versus $\mathbf{1} / \boldsymbol{R}_{\boldsymbol{d}}$ are given in Fig. A-1. The simulated results can be fitted with a straight line with a slope of 0.27 . Therefore, the surface tension at $T_{s a t}=0.86 T_{c}$ is 0.27 .

$\Delta P=\gamma / R_{d}$

Wettability of the heated surface, i.e. contact angle, could affect the pool boiling heat transfer to a great extent. In our simulation, Eq. (9) is used to calculate the solid-fluid interaction force, in which $G_{w}$ could determine the contact angle directly. In this study, $G_{w}=0$ is adopted and the intermolecular interaction force in Eq. (8) is not applied at the solid walls. The static contact angle could be determined by simulating the balance form of droplet on a horizontal surface. In this simulation, a $300 \times 100$ lattice domain is adopted and a saturated droplet with a radius of $R_{d}=40$ is placed on the bottom boundary. The buoyancy force is ignored. Periodic boundary condition is applied in $x$ direction and the no-slip boundary condition is imposed in $y$ direction. The equilibrium shape of the droplet is shown in Fig. A-2, where the contact angle is measured to be $44.5^{\circ}$.

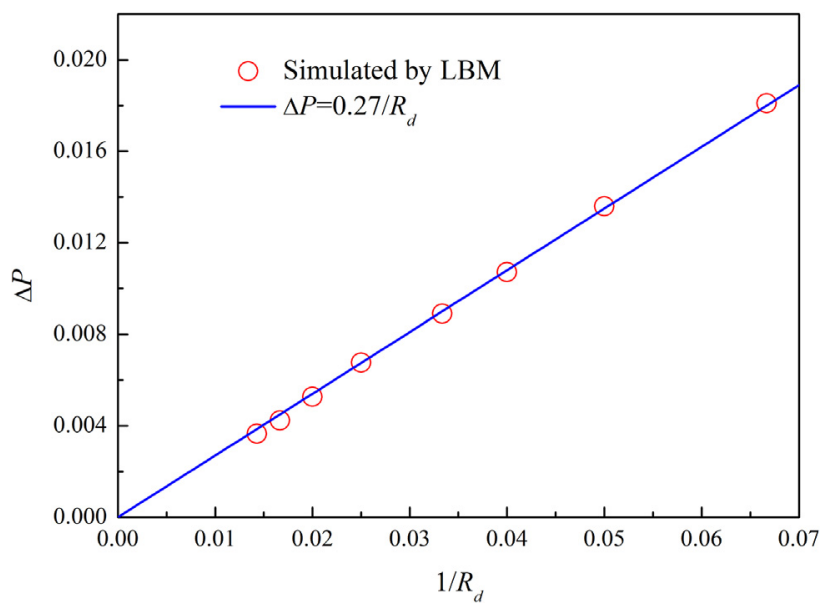

Fig. A-1. Influence of drop radius on the pressure difference of the droplet.

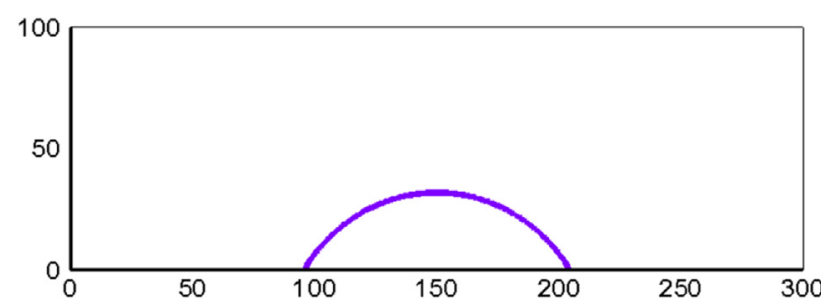

Fig. A-2. The balance form of droplet on a horizontal surface.

\section{References}

[1] V.K. Dhir, Boiling heat transfer, Annu. Rev. Fluid Mech. 30 (1) (1998) 365-401.

[2] M. Gao, P. Cheng, X. Quan, An experimental investigation on effects of an electric field on bubble growth on a small heater in pool boiling, Int. J. Heat Mass Transfer 67 (6) (2013) 984-991.

[3] M.M. Ohadi, N. Sharaf, D.A. Nelson, Electrohydrodynamic enhancement of heat transfer in a shell-and tube heat exchanger, Exp. Heat Transfer 4 (1) (1991) 1939.

[4] X. Quan, M. Gao, P. Cheng, J. Li, An experimental investigation of pool boiling heat transfer on smooth/rib surfaces under an electric field, Int. J. Heat Mass Transfer 85 (2015) 595-608.

[5] M. Uemura, S. Nishio, I. Tanasawa, Enhancement of pool boiling heat transfer by static electric field, in: Proc. 9th Internat. Heat Transfer Conf., Jerusalem, 1990, pp. 75-80.

[6] Y. Hristov, D. Zhao, D.B.R. Kenning, K. Sefiane, T.G. Karayiannis, A study of nucleate boiling and critical heat flux with EHD enhancement, Heat Mass Transfer 45 (7) (2009) 999-1017.

[7] S.W.J. Welch, G. Biswas, Direct simulation of film boiling including electrohydrodynamic forces, Phys. Fluids 19 (1) (2007) 012106.

[8] G. Tomar, G. Biswas, A. Sharma, S.W.J. Welch, Influence of electric field on saturated film boiling. Phys. Fluids 21 (3) (2009) 032107.

[9] V. Pandey, G. Biswas, A. Dalal, Saturated film boiling at various gravity levels under the influence of electrohydrodynamic forces, Phys. Fluids 29 (3) (2017) 032104.

[10] Q. Li, Q.J. Kang, M.M. Francois, Y.L. He, K.H. Luo, Lattice Boltzmann modeling of boiling heat transfer: the boiling curve and the effects of wettability, Int. J. Heat Mass Transfer 85 (2015) 787-796.

[11] Z. Dong, W. Li, Y. Song, A numerical investigation of bubble growth on and departure from a superheated wall by lattice Boltzmann method, Int. J. Heat Mass Transfer 53 (21) (2010) 4908-4916.

[12] R. Sadeghi, M.S. Shadloo, M.Y.A. Jamalabadi, A. Karimipour, A threedimensional lattice Boltzmann model for numerical investigation of bubble growth in pool boiling, Int. Commun. Heat Mass Transfer 79 (2016) 58-66.

[13] R. Sadeghi, M.S. Shadloo, Three-dimensional numerical investigation of film boiling by the lattice Boltzmann method, Numer. Heat Transfer, Part A: Appl. 71 (5) (2017) 560-574.

[14] Y. Feng, H. Li, K. Guo, J. Zhao, T. Wang, Numerical study of single bubble growth on and departure from a horizontal superheated wall by three-dimensional lattice Boltzmann method, Microgr. Sci. Technol. 30 (6) (2018) 761-773.

[15] T. Sun, W. Li, Three-dimensional numerical simulation of nucleate boiling bubble by lattice Boltzmann method, Comput. Fluids 88 (2013) 400-409.

[16] H. Safari, M.H. Rahimian, M. Krafczyk, Extended lattice Boltzmann method for numerical simulation of thermal phase change in two-phase fluid flow, Phys. Rev. E Stat. Nonlin. Soft Matter Phys. 88 (1) (2013) 013304.

[17] X. Shan, H. Chen, Lattice Boltzmann model for simulating flows with multiple phases and components, Phys. Rev. E Stat. Phys. Plasmas Fluids Relat. Interdiscipl. Top. 47 (3) (1993) 1815-1819.

[18] S. Gong, P. Cheng, Lattice Boltzmann simulations for surface wettability effects in saturated pool boiling heat transfer, Int. J. Heat Mass Transfer 85 (2015) 635-646.

[19] S. Gong, P. Cheng, Direct numerical simulations of pool boiling curves including heater's thermal responses and the effect of vapor phase's thermal conductivity, Int. Commun. Heat Mass Transfer 87 (2017) 61-71.

[20] X. Ma, P. Cheng, S. Gong, X. Quan, Mesoscale simulations of saturated pool boiling heat transfer under microgravity conditions, Int. J. Heat Mass Transfer 114 (2017) 453-457.

[21] Q. Li, P. Zhou, H.J. Yan, Improved thermal lattice Boltzmann model for simulation of liquid-vapor phase change, Phys. Rev. E 96 (2017) 063303.

[22] A.A. Mohamad, Lattice Boltzmann Method, Springer, London, 2011.

[23] Z.L. Guo, C.G. Zheng, B.C. Shi, Discrete lattice effects on the forcing term in the lattice Boltzmann method, Phys. Rev. E 65 (4 Pt 2B) (2002) 046308.

[24] Q. Li, K.H. Luo, X.J. Li, Lattice Boltzmann modeling of multiphase flows at large density ratio with an improved pseudopotential model, Phys. Rev. E Stat. Nonlin. Soft Matter Phys. 87 (5) (2013) 053301.

[25] I. Ginzburg, P.M. Adler, Boundary flow condition analysis for the threedimensional lattice Bolzmann model, J. Phys. II 4 (2) (1994) 191-214.

[26] G. Hazi, A. Markus, On the bubble departure diameter and release frequency based on numerical simulation results, Int. J. Heat Mass Transfer 52 (5) (2009) $1472-1480$. 
[27] L.D. Landau, E.M. Lifshitz, Electrodynamics of Continuous Media, Pergamon Press Inc., Oxford, 1960.

[28] X. He, N. Li, Lattice Boltzmann simulation of electrochemical systems, Comput. Phys. Commun. 129 (1) (2000) 158-166.

[29] J. Zhang, D.Y. Kwok, A 2D lattice Boltzmann study on electrohydrodynamic drop deformation with the leaky dielectric theory, J. Comput. Phys. 206 (1) (2005) 150-161.

[30] A.L. Kupershtokh, D.A. Medvedev, Lattice Boltzmann equation method in electrohydrodynamic problems, J. Electrostat. 64 (7) (2006) 581-585.

[31] S. Gong, P. Cheng, X. Quan, Lattice Boltzmann simulation of droplet formation in microchannels under an electric field, Int. J. Heat Mass Transfer 53 (25-26) (2010) 5863-5870.

[32] N. Wang, Q. Lei, Lattice Boltzmann model for simulation of avalanche formation and streamer discharge in breakdown of gaseous dielectrics, Appl. Comput. Electromagn. Soc. J. 30 (10) (2015) 1129-1136.

[33] W.F. Huang, Y. Li, Q.S. Liu, Application of the lattice Boltzmann method to electrohydrodynamics: deformation and instability of liquid drops in electrostatic fields, Chin. Sci. Bull. 52 (24) (2007) 3319-3324.

[34] C.K. Law, Recent advances in droplet vaporization and combustion, Prog. Energy Combust. Sci. 8 (3) (1982) 171-201.

[35] M.S. Shadloo, A. Rahmat, M.J.C.M. Yildiz, A smoothed particle hydrodynamics study on the electrohydrodynamic deformation of a droplet suspended in a neutrally buoyant Newtonian fluid, Comput. Mech. 52 (3) (2013) 693-707.
[36] J.D. Sherwood, Breakup of fluid droplets in electric and magnetic fields, J. Fluid Mech. 188 (1988) 133-146.

[37] Y. Wang, D. Sun, A. Zhang, B. Yu, Numerical simulation of bubble dynamics in the gravitational and uniform electric fields, Numer. Heat Transfer Part A: Appl. 71 (10) (2017) 1034-1051.

[38] L. Yuan, Two-phase electro-hydrodynamic flow modeling by a conservative level set model, Electrophoresis 34 (5) (2013) 736-744.

[39] J. Hua, K.L. Liang, C.H. Wang, Numerical simulation of deformation/motion of a drop suspended in viscous liquids under influence of steady electric fields, Phys. Fluids 20 (11) (2008) 295.

[40] S. Gong, P. Cheng, Lattice Boltzmann simulation of periodic bubble nucleation, growth and departure from a heated surface in pool boiling, Int. J. Heat Mass Transfer 64 (3) (2013) 122-132.

[41] V. Pandey, G. Biswas, A. Dalal, Effect of superheat and electric field on saturated film boiling, Phys. Fluids 28 (5) (2016) 052102.

[42] J. Berghmans, Electrostatic fields and the maximum heat flux, Int. J. Heat Mass Transfer 19 (7) (1976) 791-797.

[43] R. Johnson, Effect of an electric field on boiling heat transfer, AIAA J. 6 (8) (1968) 1456-1460. 